osteoarthritis $(\mathrm{OA})$ is to unload the affected compartment, this is accomplished by correcting the angular deformity towards the unaffected compartment, i.e. shifting the hip-knee-ankle angle (HKA; mechanical axis) towards varus for a medial lesion. Knee joint distraction (KJD) is an alternative joint-sparing treatment for knee $\mathrm{OA}$ and has been demonstrated to decrease pain, improve function, and increase joint space width (JSW) ${ }^{1}$.

Objectives: To investigate the importance of axial alignment (and correction) in these two effective (joint-sparing) treatments of medial knee OA.

Methods: Patients with medial knee OA, a HKA less than $12^{\circ}$ varus, normal knee stability, younger than 65 years, and a BMI less than $35 \mathrm{~kg} / \mathrm{m}^{2}$ were randomized to HTO $(n=46)$ or KJD $(n=23)$. WOMAC and VAS pain were collected at baseline and after twelve months. To assess structural outcome, JSW was measured on knee radiographs, before and after both treatments. HTO patients had full leg standing anteroposterior radiographs taken before and after surgery, KJD patients only had these taken before surgery. Therefore, the femur-tibia angle (FTA; anatomical axis), acquired using Knee Image Data Analysis (KIDA), was investigated as an alternative for assessing axial alignment. Agreement between axial alignment as defined by HKA and by FTA appeared to be fair (ICC $=-0.414)$. WOMAC and VAS Pain were then related to (changes in) axial alignment, Kellgren \& Lawrence (K\&L) grade, BMI, gender, pre-operative range of motion (ROM), and age as independent variables in linear regression models.

Results: Patient baseline characteristics were not statistically significantly different between patients treated with KJD or HTO (see table 1). WOMAC increased statistically significantly one year after either treatment (KJD: $\triangle 21.05 \pm 19.93$; HTO: $\triangle 27.80 \pm 15.32$; both $p<0.001$ ). Likewise, VAS pain decreased (KJD: $\Delta-23.89 \pm 29.67, p=0.001 ; H T O: \Delta-35.42 \pm 24.06, p<0.001)$. KJD led to a statistically significant increase in mean JSW $(\Delta 0.50 \pm 0.88 \mathrm{~mm}, \mathrm{p}=0.014)$, and both treatments led to a statistically significant increase in medial (KJD: $\Delta 0.81 \pm 1.16 \mathrm{~mm}, \mathrm{p}=0.004$; HTO: $\Delta 0.47 \pm 0.69 \mathrm{~mm}, \mathrm{p}<0.000$ ) as well as $\mathrm{min}$ imal JSW (KJD: $\Delta 0.85 \pm 0.96 \mathrm{~mm}, \mathrm{p}<0.000$; HTO: $\Delta 0.35 \pm 0.51 \mathrm{~mm}, \mathrm{p}<0.000$ ) after one year. The FTA changed significantly in the HTO group after one year $\left(\Delta 0.73^{\circ}, \mathrm{p}=0.005\right)$, while the $\mathrm{KJD}$ group showed a trend $\left(\Delta 0.77^{\circ}, \mathrm{p}=0.105\right)$. In the KJD group, changes in clinical outcomes were not associated with pre-operative HKA, changes in FTA, K\&L grade, BMI, gender, pre-operative ROM, or age. In contrast, in the HTO group a significant association was demonstrated for a change in WOMAC with a change in FTA (std. $\beta=-0.341)$ and for a change in VAS Pain with baseline age (std. $\beta=-0.323$ ), as seen in table 2 .

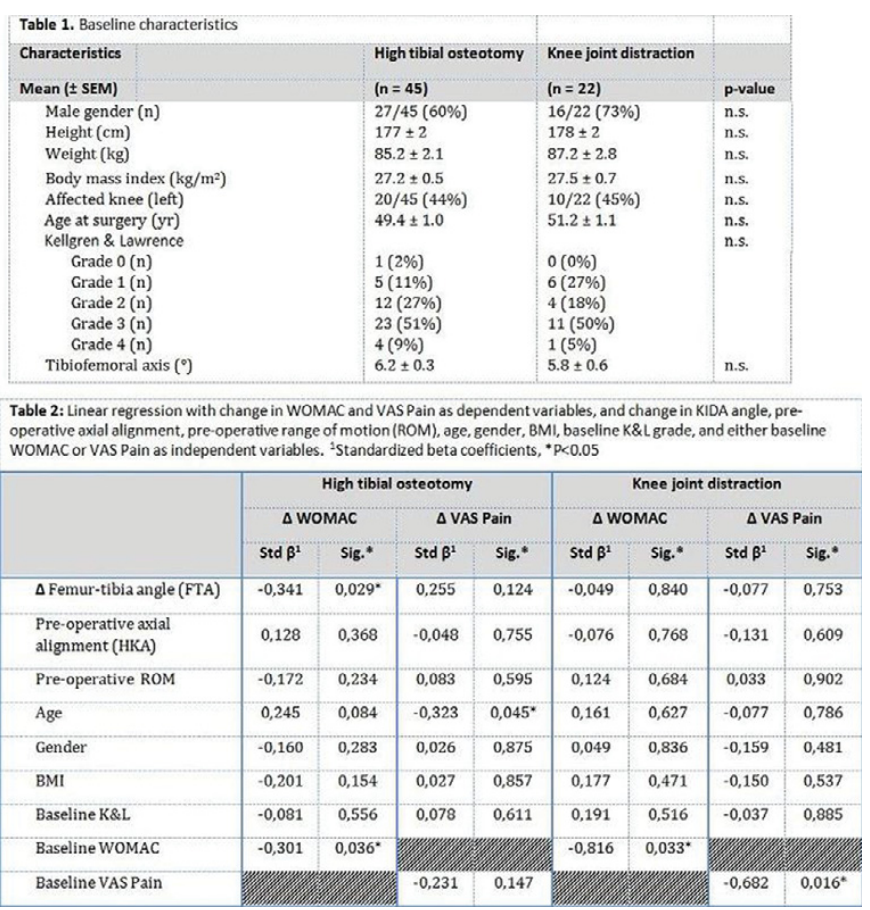

Conclusions: Both KJD and HTO lead to a statistically significant clinical and structural benefit after one year. Nevertheless, the change in FTA was associated with WOMAC change after one year in the HTO group, but not in the KJD group. This indicates that axial alignment correction may not per se be necessary for clinical benefit.

References:

[1] van der Woude et al. Five-Year Follow-up of Knee Joint Distraction. 2016 Cartilage.

Disclosure of Interest: None declared

DOI: 10.1136/annrheumdis-2017-eular.3363

\section{SAT0537 INFLUENCE OF MELOXICAM IN ORODISPERSIBLE FORM ON PLATELET AGGREGATION AND VON WILLEBRAND FACTOR IN PATIENTS WITH OSTEOARTHRITIS}

O.S. Khmel, V.V. Rodionova. Occupational Diseases and Clinical Immunology, State Establishment "Dnipropetrovsk Medical Academy, of Health Ministry of Ukraine", Dnepr, Ukraine

Background: Meloxicam, which selectively inhibits COX-2, can cause inhibition of the biosynthesis of vascular endothelium vasodilator - prostacyclin, without impacting significantly on production of thromboxane, which promotes vasoconstriction [1]. Therefore, the effect of Meloxicam on the possibility of thrombotic complications need to be learn more accurately.

Objectives: To investigate the effect of orodispersible form of Meloxicam on platelet aggregation and von Willebrand factor in patients with knee osteoarthritis. Methods: The study included 24 patients with knee osteoarthritis $(\mathrm{OA})$ of the II stage according to the Kellgren-Lawrence. The control group consisted of 15 healthy individuals. Patients were prescribed the orodispersible form of Meloxicam in dose of $15 \mathrm{mg} 1$ time per day orally during 10 days. The survey was carried out before and after treatment. Patients had all-clinical studies, questionnaires (visual analogue scale (VAS), Western Ontario and McMaster Universities Arthritis Index (WOMAC), questionnaire Lequesne), optical aggregometry with adenosine diphosphate (ADP), collagen, thrombin and ristocetin for revealing the level of von Willebrand factor.

Results: As a result of treatment patients had a significant improvement of overall health and reduction of pain in knee joints according to the VAS (before treatment - $54.5[50-71] \mathrm{mm}$, after treatment $-27[18-41] \mathrm{mm} ; \mathrm{p} \leq 0.05)$, WOMAC (before treatment $-143[109-187]$ points, after treatment - 98 [13-168] points; $p \leq 0.05)$, questionnaire Lequesne (before treatment - 16 [13 - 21] points, after treatment $-12[3-22]$ points; $p \leq 0.05)$. After treatment patients experienced a significant increase in the degree of platelet aggregation with ADP (before treatment $-52.6[39.6-98.2] \%$ after the treatment $-83.5[41.3-127] \% ; p \leq 0.05)$, which may indicate a probable increase in the initiation of irreversible aggregation of circulating platelets. The degree of platelet aggregation with collagen also increased (before treatment $-46.5[29.5-89] \%$, after treatment - $68.6[37.9-$ $115.4] \% ; p<0.05$ ), indicating the increased adhesion of platelets to collagen of the vascular endothelium. Before and after treatment, patients remained significantly elevated degree of aggregation with thrombin in comparison with the control group (before treatment - 65.6 [24.7 - 86.7], after treatment - 78 [62.3 - 92.7]\%, control group - $37.8[32.11-42.26] \% ; p \leq 0.05$ ) which indicates the stimulation of the of the endothelin-1 synthesis with further infringements of procoagulants and anticoagulants. Von Willebrand factor, as an indirect indicator of endothelial damage, was significantly increased after treatment (before treatment - 151.4 $[138.9-224] \%$ after treatment $-206.8[171.9-257.4] \%)$, which may indicate increase of endothelial lesions because of meloxicam with further endothelial dysfunction $(p \leq 0.05)$

Conclusions: Intake of the orodyspersible form of Meloxicam in patients with osteoarthritis can cause an increase of platelet aggregation and level of von Willebrand factor that may contribute to the vascular endothelial dysfunction and increase in risk of thrombosis.

\section{References:}

[1] Wittenauer R., Smith L., Aden K. Update on 2004 Background Paper Written by Saloni Tanna, Pharm.D. MPH Background Paper 6.12 Osteoarthritis. Priority Medicines for Europe and the World "A Public Health Approach to Innovation"; 2013. P. 31.

Disclosure of Interest: None declared

DOI: 10.1136/annrheumdis-2017-eular.5348

\section{SAT0538 PROGRESSION OF PAIN, NUMBER OF CLINICALLY SWOLLEN JOINTS AND ULTRASOUND DETECTED SYNOVITIS AND OSTEOPHYTE FORMATION IN PATIENTS WITH HAND OSTEOARTHRITIS OVER TWO YEARS}

O. Sleglova, O. Ruzickova, K. Pavelka, L. Senolt. Institute of Rheumatology, Prague, Czech Republic

Background: Hand osteoarthritis (HOA) is a common and frequent cause of pain. $\mathrm{HOA}$ is a heterogeneous group of disorders with two main subsets including nonerosive and erosive disease. Few studies demonstrated inflammatory ultrasound changes and more severe clinical symptoms in patients with erosive compared with non-erosive disease, however the results are inconsistent.

Objectives: he aim of this study was to evaluate progression of pain, stiffness, physical impairment and ultrasound features in patients with erosive and nonerosive HOA in a two years longitudinal study.

Methods: Consecutive patients with symptomatic HOA fulfilling the American College of Rheumatology (ACR) criteria were included in this study. Joint pain and swelling were assessed. Patients reported joint pain on $100 \mathrm{~mm}$ visual analogue scale (VAS). Pain, joint stiffness and disability were assessed by the Australian/Canadian OA hand index (AUSCAN). Radiographs of both hands were examined and erosive disease was defined by at least one erosive interphalangeal joint. Synovial hypertrophy and power Doppler signal (PDS) were scored with ultrasound. Synovitis was graded on a scale of $0-3$ and osteophytes were defined as cortical protrusions seen in two planes. Patients were examined at baseline and at the two years follow-up. 
Results: Altogether, 129 patients (12 male) with symptomatic nodal HOA were included in this study and followed between April 2012 and January 2017. Out of these patients, 72 had erosive disease. The disease duration $(p<0.01)$ was significantly higher in patients with erosive compared with non-erosive disease. Pain $(p<0.05)$ and the number of clinically swollen joints $(p<0.05)$ were significantly higher in patients with erosive compared with non-erosive disease at baseline. There were significant progression of pain $(p<0.05)$ and the number of clinically swollen joints $(p<0.01)$ at the two years follow up. The progression in clinically swollen joint count was about $21 \%$ higher in patients with erosive disease.

According to the AUSCAN, patients with erosive compared with non-erosive disease had more pain $(p<0.05)$ and stiffness $(p<0.01)$ at baseline. Pain and stiffness, but not function, got worse $(p<0.01)$ in patients with erosive compared with non-erosive disease at the two years follow up.

US-detected pathologies such as gray-scale synovitis total score $(p<0.001)$, intensity of PDS $(p<0.01)$ and number of osteophytes $(p<0.01)$ were significantly higher in patients with erosive compared with non-erosive disease at baseline. There were improvements in gray-scale synovitis total score and intensity of PDS in patients with non-erosive disease while patients with erosive disease worsened at the two years follow up. On the other hand, the progression of US-determined osteophyte formation was observed in both groups.

Conclusions: The findings of this study show that pain and number of clinically swollen joints associated with US-detected synovial hypertrophy inflammatory signs and osteophyte formation is more severe in patients with erosive HOA than in patients with non-erosive disease. In addition, osteophyte formation is more likely to progress independent of synovial inflammation.

Acknowledgements: This work was supported by the project (Ministry of Health, Czech Republic) for consensual development of research organization 023728. Disclosure of Interest: None declared

DOI: 10.1136/annrheumdis-2017-eular.2795

\section{SAT0539 DIFFERENTIAL PROFILE OF ENDOGENOUS PEPTIDES DETECTED BY TARGETED PROTEOMICS IN HIP AND KNEE CARTILAGE SECRETOMES FROM OSTEOARTHRITIS PATIENTS}

P. Fernandez-Puente, L. Gonzalez-Rodriguez, V. Calamia, L. Lourido, M. Camacho, C. Ruiz-Romero, F.J. Blanco. Grupo de

Proteomica-PBR2-ProteoRed/ISCIII-Servicio de Reumatología., Instituto de Investigación Biomédica de A Coruña (INIBIC), Complexo Hospitalario Universitario de A Coruña (CHUAC), Sergas. Universidade da Coruña (UDC), A Coruña, Spain

Background: Peptidomics can be defined as the comprehensive, qualitative and quantitative study of all native peptides in a sample in a defined space at a defined time under defined biological conditions. LC-MS/MS targeted proteomics have been used to identify and quantify candidate molecular biomarkers in diverse range of samples, including cells, tissues, serum/plasma, and other types of body fluids.

Objectives: In the present work, we have developed a targeted method to detect a panel of endogenous peptides that were identified as released from human healthy $(\mathrm{N})$ and/or osteoarthritic $(\mathrm{OA})$ hip and knee cartilage. Secretomes were obtained separately from the wounded (WZ) and unwounded (UZ) zones of OA cartilage. The enrichment of endogenous peptides was achieved by ultrafiltration and solid phase extraction (SPE). After peptide extraction, the different peptide profiles of these secretomes were relative quantified by target proteomics.

Methods: Proteins secreted from human articular cartilage (secretomes) were obtained by culture of tissue explants [1]. The enrichment of endogenous peptides was standardized using ultrafiltration procedures and solid phase extraction with reversed phase $(\mathrm{C} 18)$ resins. A method for the targeted identification and relative quantitation of endogenous peptides by Multiple Reaction Monitoring (MRM)-mass spectrometry was developed employing cartilage secretome samples, and then applied on synovial fluid and serum. The peptides were separated by nano-LC coupled to a 5500 QTRAP mass spectrometer using stable isotope-labeled (SI) peptides as an internal control. Peptide identifications were searched using the ProteinPilot program. Data analysis was performed using the Skyline software.

Results: 23 endogenous peptides belonging to 9 proteins related with $\mathrm{OA}$, as Cartilage Oligemeric Matrix Protein (COMP), Cartilage Intermediate Layer Protein (CILP), Prolargin (PRELP), Dermcidin (DCD), Fibronectin (FINC) and Glia Derived Nexin (GDN) among others, were differentially detected and relatively quantified in the cartilage secretomes. Some of the endogenous peptides belonging to COMP, FINC and GDN were found with a significant high release in the UZ of hip cartilage, however in knee cartilage the release is higher in the WZ. On the other hand, certain peptides belonging to proteins like CILP or PRELP were found to be mostly increased in the UZ of both hip and knee OA cartilages when compared to healthy tissue.

Conclusions: A panel of endogenous peptides has been identified in articular cartilage, which are differentially released between OA and healthy patients, as well as between knee and hip OA patients. These endogenous peptides may be potential biomarkers to differentiate osteoarthritis from hip and knee. Our targeted proteomics approach would be widely applicable to quantify low abundant peptides of interest in complex biological samples as synovial fluid and serum in order to unravel their putative biomarker value for osteoarthritis.
References:

[1] Lucía Lourido et al. "Quantitative Proteomic Profiling of Human Articular Cartilage Degradation in Osteoarthritis". J Proteome Res. 2014 Dec 5;13(12):6096-106.

Disclosure of Interest: None declared

DOI: 10.1136/annrheumdis-2017-eular.6185

\section{SAT0540 THE REDUCTION IN ADRENERGIC AND INFLAMMATORY SERUM MARKERS IN OSTEOARTHRITIS PATIENTS AFTER TREATMENT WITH CHONDROITIN SULFATE/GLUCOSAMINE HYDROCHLORIDE AND CELECOXIB IS DIFFERENT ACCORDING TO THE PRESENCE OF BACTERIAL DNA IN BLOOD}

P. Zapater $^{1}$, M. Herrero ${ }^{2}$, H. Martínez ${ }^{2}$, R. Francés ${ }^{3,4}$, J. Vergés ${ }^{5}$, J.F. Horga ${ }^{6}$ ${ }^{1}$ Pharmacology Department, Miguel Hernández University, Alicante; ${ }^{2}$ Clinical R\&D, Bioiberica, Barcelona; ${ }^{3}$ CIBERehd. Instituto de Salud Carlos III, Madrid; ${ }^{4}$ Medicine Department, Miguel Hernández University, Alicante; ${ }^{5}$ Osteoarthritis Foundation International (OAFI), Barcelona; ${ }^{6}$ Pharmacology Department, Miguel Hernández University, Alicante, Spain

Background: Inflammation in osteoarthritis (OA) has been characterized by infiltration of immune cells and secretion of cytokines into synovial tissues. Synovial macrophages production of IL-1beta and TNF-alfa is increased in response to lipopolysaccharide (LPS) suggesting a potential role of pathogenassociated molecular patterns (PAMPs) in OA development and/or evolution. Adrenergic activity have been associated with subchondral bone loss and increased osteoclast activity. Also, adrenergic activity is a main modulator of immune response to PAMPs in different settings. However,the relationship between activation of adrenergic and immune system with OA progression and treatment response remains still unknown.

Objectives: To assess the adrenergic and immune activation in OA patients according to the presence of bacterial DNA (bDNA) fragments in blood before and after Chondroitin Sulfate/Glucosamine Hydrochloride (CS/GH) or Celecoxib (CE) treatment.

Methods: Serum samples from patients participants in a 6-month controlled, double blind and randomized clinical trial comparing the analgesic efficacy of $\mathrm{CS} / \mathrm{GH}$ and CE were analyzed to determine cytokines (IL-2, IL-4, IL-6, IL-10, IL-8, IL-1beta), catecholamines (noradrenaline, adrenaline and dopamine) and the presence of endotoxin or LPS and bDNA. Results are shown as mean \pm sd or median with ranges (Q25-Q75).

Results: Samples from 100 OA patients (age: $62 \pm 8 \mathrm{yr}$.; BMI: $31 \pm 6 \mathrm{~kg} / \mathrm{m}^{2} 86$ females; VAS: $73 \pm 15$; WOMAC: $369 \pm 43$ ) treated with CS/GH (50) or CE (50) were analyzed. There were no baseline significant differences between the two treatment groups regarding demographics,clinical and experimental variables. Thirty-four patients (17-CS/GH and 17-CE) showing bDNA in blood had significantly higher levels of noradrenaline compared with patients without bDNA (1993 [1354-3183] vs. 1324 [738-1899]; $p=0.0002$ ). After 6 months, both groups of treatment showed a similar reduction in VAS and WOMAC score (pain assessment) and serum adrenaline levels independent of presence or absence of bDNA (Table 1). Thirtythree patients showed bDNA presence at the end of the study ( 23 negative and 10 positive at baseline). Patients with bDNA at 6 months after treatment showed reduced serum noradrenaline levels compared with those observed in patients with bDNA at baseline (1993 [1354-3183] vs. 1561 [1174-2193]; $p=0.0325)$. IL-8 was significantly reduced after 6 months of treatment only in patients without bDNA.There were no significant differences between baseline and 6 months samples in the other experimental variables.

\section{Table 1}

\begin{tabular}{|c|c|c|c|c|c|c|c|c|}
\hline & \multicolumn{4}{|c|}{ Baseline } & \multicolumn{4}{|c|}{6 months } \\
\hline & \multicolumn{2}{|c|}{ No bONA } & \multicolumn{2}{|c|}{ bDNA } & \multicolumn{2}{|c|}{ No bONA } & \multicolumn{2}{|c|}{ bONA } \\
\hline & CS/GH & CE & $\mathrm{CS} / \mathrm{GH}$ & $\mathrm{CE}$ & $\mathrm{CS} / \mathrm{GH}$ & $C E$ & $\mathrm{CS} / \mathrm{GH}$ & $\mathrm{CE}$ \\
\hline Adrenaline & 98 & 100 & 92 & 98 & $78^{*}$ & $81^{\circ}$ & $76^{*}$ & $77^{\circ}$ \\
\hline$(\mathrm{pg} / \mathrm{ml})$ & $(88: 127)$ & (77.118) & (78:114) & $(75-115)$ & (65.92) & $(62.92)$ & (58.97) & $(66-105)$ \\
\hline Noradrenaline & 1357 & 11228 & 1723\# & $2765 \#$ & 1050 & \begin{tabular}{|l|}
1588 \\
\end{tabular} & 1563 & 1528 \\
\hline (pgs/mL) & (790-1693) & $(738-1967)$ & (1385-2381] & $(12322-3250)$ & (811-1600) & $\mid(882-2133 \mid$ & $(1209 \cdot 2180)$ & (1174-2229) \\
\hline |1.-8(p//ml) & $\begin{array}{c}5.5 \\
(1.3 .9 .4)\end{array}$ & $\begin{array}{c}5.6 \\
(1.7-14)\end{array}$ & $\begin{array}{c}4.8 \\
(3.9 .5 .7)\end{array}$ & $\begin{array}{c}11 \\
(19 .-15)\end{array}$ & $\begin{array}{c}3.3^{*} \\
(0.0-11)\end{array}$ & $\begin{array}{c}0.0^{*} \\
(0.0 .5 .9)\end{array}$ & $\begin{array}{c}6.1 \\
(3.7-14)\end{array}$ & $\begin{array}{c}5.6 \\
(1.5-16)\end{array}$ \\
\hline VAS score & 72 & 75 & 79 & 76 & $32^{*}$ & $20^{*}$ & $37^{*}$ & $52^{*}$ \\
\hline$(0-100)$ & $(66-82)$ & $(655-81)$ & $(67-90)$ & $(70-80)$ & $(22-45)$ & $(10-60)$ & (27.50) & $(24-60)$ \\
\hline WOMAC score & $\begin{array}{c}364 \\
(341-389)\end{array}$ & $\begin{array}{c}354 \\
(321-389)\end{array}$ & $\begin{array}{c}382 \\
(372-407)\end{array}$ & $\begin{array}{c}360 \\
(340-400)\end{array}$ & $\begin{array}{c}150^{*} \\
(87 \cdot 217)\end{array}$ & $\begin{array}{l}135^{*} \\
(64-238)\end{array}$ & $\begin{array}{c}180^{*} \\
(842218)\end{array}$ & $\begin{array}{c}282^{*} \\
(139 \cdot 318)\end{array}$ \\
\hline
\end{tabular}

Conclusions: OA patients show bDNA fragments in blood associated to higher serum noradrenaline levels. Six month treatment with $\mathrm{CS} / \mathrm{GH}$ or CE reduces significantly and in a similar fashion the pain intensity and adrenaline levels. IL-8 levels were reduced with the treatment except in patients with presence of bDNA fragments in blood. Systemic adrenergic and inflammatory activity in OA patients is influenced by the presence of PAMPs as bDNA in blood and this may be a factor to take into consideration to evaluate the severity, evolution and response to treatments. 